\title{
Le personnage féminin et la lecture du temps dans Le Premier Jardin d'Anne Hébert
}

\author{
Alex Noël \\ Université McGill
}

L'histoire du roman moderne québécois est particulière. La plupart des romanciers bénéficiant d'une reconnaissance à l'extérieur du champ littéraire québécois sont en fait des romancières (Gabrielle Roy, Anne Hébert, Marie-Claire Blais, Antonine Maillet ${ }^{1}$ ). Toutefois, s'il ne venait à personne l'idée de douter qu'un écrivain comme Réjean Ducharme ait bel et bien

\footnotetext{
1 Anne Hébert, Marie-Claire Blais, Gabrielle Roy et Antonine Maillet ont respectivement remporté les prix Femina, Médicis, Femina et Goncourt, qui comptent parmi les distinctions littéraires les plus prestigieuses de la francophonie.
} 
écrit une œuvre qui participe du genre romanesque - malgré certaines transgressions formelles -, la critique a beaucoup douté de la capacité de Gabrielle Roy et d'Anne Hébert à écrire des romans. L'œuvre de Gabrielle Roy a en effet souvent été rangée parmi les recueils de nouvelles et les autobiographies. Dans le cas d'Anne Hébert, sous prétexte que son écriture est plus poétique que prosaïque et que la forme a préséance sur le contenu, certains théoriciens ont préféré qualifier ses romans de récits poétiques.

Il convient peut-être, en guise d'introduction, de rappeler l'un des fils conducteurs du genre romanesque, soit le parcours existentiel du personnage. Cela nous permettra de camper notre sujet et d'exposer le rapport au temps qu'entretient le personnage féminin hébertien, en s'appuyant sur le cas de Flora Fontanges, l'héroïne du Premier Jardin. Comme le souligne Isabelle Daunais dans son essai sur la mémoire romanesque intitulé Les Grandes Disparitions, presque tous les grands personnages de roman suivent un parcours ontologique précis : ils s'aventurent dans le monde contemporain qui leur est étranger en conservant la mémoire d'un monde disparu, monde auquel seul ils appartiennent. En cela résiderait l'ontologie du personnage romanesque :

une fois constatée la disparition du monde qu'il considère sien, le personnage romanesque doit affronter le fait que la vie ne cesse pas de se poursuivre et le temps de s'écouler. Or pour décider d'une conduite à tenir, pour avancer dans ce temps, il n'a pas d'autre choix que de recourir aux souvenirs qu'il a emportés avec lui et qui constituent son trésor. Le personnage le plus audacieux comme le plus désillusionné n'avance jamais que dans ce lien paradoxal avec le passé, qui certes le guide, étant le seul outil en sa possession pour déchiffrer le monde, mais aussi le leurre, en lui donnant pour référence et pour modèle ce qui n'existe plus. (Daunais, p. 24) 
Don Quichotte, pour prendre un exemple bien connu, s'aventure dans le monde moderne (symbolisé par les marchands) alors qu'il appartient en tous points au monde médiéval chevaleresque; Emma Bovary conserve quant à elle la mémoire du romantisme dans un monde associé à la bourgeoisie médiocre; même Joseph K., le héros du Procès, s'aventure dans une société arbitraire avec le souvenir d'un monde où règnent la justice et l'ordre social. Ce décalage entre le monde contemporain et le monde auquel il croit appartenir serait, selon Isabelle Daunais, un trait caractéristique du personnage romanesque.

\section{Flora Fontanges et la disparition de la ville ancienne}

Pris sous cet angle, le caractère romanesque d'une œuvre comme Le Premier Jardin d'Anne Hébert paraît indéniable. Le personnage de Flora Fontanges, une comédienne, décide de quitter la France après plusieurs années d'exil et de rentrer dans sa ville natale de Québec à la suite de la disparition de sa fille et de l'offre qu'on lui fait de jouer Winnie dans Oh les beaux jours de Beckett. Or, le personnage de Flora suit un parcours similaire à celui de Don Quichotte et d'Emma Bovary. En compagnie du jeune Raphaël, un ami de sa fille qui étudie en histoire, Flora Fontanges marche dans les rues de Québec avec la mémoire d'une ville disparue. D'abord, elle constate que le paysage visuel de son enfance, tel qu'elle l'avait connu, a été transformé par le temps :

Elle regarde les maisons victoriennes, transformées en cafés et en restaurants. Elle se demande quand est-ce que cela a commencé, tous ces parasols, ces marquises bariolées, ces 
petites tables, ces chaises plantées comme sur une plage, tout le long de la Grande-Allée.

[...] Touristes et fonctionnaires de passage déambulent le long des trottoirs. Font trois petits tours et puis s'en vont. Ne sont pas nés ici. Ne mourront pas ici. Tout juste de passage. [...] Mais où sont les gens? Les vrais. Ceux qui ont eu vie liée avec les boiseries sombres, les sous-sols incommodes, les escaliers tuants, les étages empilés, les cheminées ronflantes. Se sont-ils retirés, dorment-ils de leur dernier sommeil, murés dans la pierre de taille de leurs demeures, aux larges bow windows? (Hébert, p. 21)

Le parcours de Flora Fontanges est contraire au héros homérique : Ulysse, revenant d'un exil d'une vingtaine d'années, retrouve son Ithaque natale qui l'attend sous la figure de Pénélope. Autrement dit, le monde qu'il avait connu lui est redonné. Étrange situation donc que celle de Flora Fontanges qui, revenant d'un exil de plusieurs années, découvre que toutes les personnes qu'elle a connues dans son enfance, la rue où habitait sa famille adoptive, l'apparence des maisons, les gens qui habitaient la ville ont tous et toutes disparu. En fait, Flora Fontanges se pose «la question que le roman n'a jamais cessé de se poser [...], c'est-à-dire "que se passe-t-il lorsque le monde dont on est issu et que l'on reconnaît seul pour sien est un monde disparu?" » (Daunais, p. 9)

En fait, Flora «marche dans les rues qui lui paraissent étrangères » (Hébert, p. 21) et, tout comme Don Quichotte qui tente de ressusciter la chevalerie dans le monde empirique, elle tente de «faire venir le temps d'autrefois sur la ville » (p. 86) :

depuis longtemps, Flora Fontanges est persuadée que, si un jour, on arrive à tout rassembler du temps révolu, tout, exactement tout, avec les détails les plus précis - air, heure, lumière, température, couleurs, textures, odeurs, objets, meubles - on doit parvenir à revivre l'instant passé dans toute sa fraîcheur. (p. 104) 
Flora Fontanges marche dans les rues de la ville moderne de Québec en évoquant le passé de Québec: les Filles du roi, les premiers arrivants, les Amérindiens, etc. Cette vision passéiste de la ville semble même occulter la ville contemporaine, qui ne sert plus que de relais mémoriel à la ville du passé. Plus encore, la comédienne sélectionne son parcours géographique de manière à éviter «la ville nouvelle, moitié village, moitié banlieue, [qui] entoure la ville ancienne comme une ceinture verte » (p. 98).

\section{Flora Fontanges et Raphaël : deux lectures du temps}

Toutefois, contrairement à Emma Bovary ou à Joseph K, Flora Fontanges n'est pas livrée seule à sa quête : elle est en effet accompagnée du jeune Raphaël. Aussi cette promenade dans la ville est-elle l'occasion, pour la romancière, de suggérer que le personnage masculin de Raphaël, lié à l'historiographie par son métier de guide et ses études en histoire, et le personnage féminin de Flora (représentatif des personnages féminins d'Anne Hébert), une comédienne, possèdent deux manières différentes de lire et d'interpréter le passé.

À cet effet, tout au long du Premier Jardin, le champ lexical de l'Histoire (ou du rapport au passé) diffère d'un personnage à l'autre. D'une part, Raphaël est «étudiant en histoire à l'université » (Hébert, p. 18) et "raconte la ville aux touristes » (p. 18). Il entraîne Flora Fontanges dans le « circuit habituel. La maison de Montcalm, les trésors des ursulines et de l'HôtelDieu» (p. 29). De plus, «Raphaël, en bon guide attitré, a emporté un plan de la ville» (p.37). Il «énumèr[e] des noms 
d'églises au passage » (p. 41), lui présente des «maquettes bien alignées» (p. 29) et lui «parle du musée» (p.52) et «des fortifications qu'on a mis cent ans à ériger» (p. 55). C'est là ce que le narrateur nomme, à juste titre, «la version de Raphaël » (p. 37). Sa manière à lui, très canonique, de lire le passé est rationnelle, organisée, scientifique. Le passé est quelque chose d'extérieur à lui, d'achevé, c'est-à-dire avec un commencement, un ordre chronologique et une fin.

La version de Flora Fontanges, sans être opposée à celle $\mathrm{du}$ jeune étudiant, est du moins complètement différente. Si Flora évoque le passé, c'est avant tout dans le but «de s'approprier la ville» (Hébert, p. 120). D’abord, elle entretient un rapport personnel avec le passé de Québec qui lui évoque son propre passé. Mais surtout, elle se sent dans la ville comme dans un « décor de théâtre » (p. 49) dans lequel « [e]lle cherche un nom de femme à habiter» (p. 49). En fait, sa mémoire est aussi une vengeance contre les oublis de l'histoire. Elle cherche à s'approprier les personnages de femmes du passé; elle aime, par exemple, à se répéter le nom des Filles du roi, à imaginer leur vie, à interpréter leur rôle, pour les rendre vivantes à nouveau, pour les venger de l'histoire morte. Sa "version » est donc très différente de celle de Raphaël, moins désintéressée, voire personnalisée. Elle cherche à faire revivre le passé par le biais du théâtre pour accéder à un symbolisme universel, c'està-dire que, contrairement à Raphaël, le passé, pour Flora Fontanges n'est pas un objet mort, soit une représentation figée du temps, mais se trouve au contraire réactualisé dans le présent.

Le «cas» de Barbe Abbadie est révélateur de la différence, mais aussi de la complémentarité des deux lectures 
historiques proposées par la romancière. En marchant dans les rues avoisinant la place Royale, en basse-ville, Flora Fontanges découvre que, contrairement à ce que lui avait affirmé Raphaël, aucune de ces rues ne porte le nom de Barbe Abbadie. Flora se demande donc si, à partir de son seul nom, elle peut retrouver la femme qui le portait, en remplissant de sens le sème vide que constitue un nom propre. Par ailleurs, c'est bien ce que fait Flora Fontanges qui, «tout au long de sa carrière, [...] s'est laissé prendre par des titres de pièces qui étaient des noms de femmes, alors qu'elle ne savait rien encore du texte à suivre. Des noms pour rêver et mûrir un rôle en secret, avant que les répliques ne surgissent» (Hébert, p.50). Or, Raphaël, qui la rejoint en cours de route, plutôt que de s'opposer à son projet, « entre dans le jeu » (p. 51) de Flora et y prend goût. Ils parlent tous les deux de Barbe Abbadie, «décident d'un commun accord de l'âge de Barbe Abbadie, de son état civil, de sa vie et de sa mort » (p. 51). Toutefois, Raphaël est celui qui cherche à «situer l'époque à laquelle Barbe Abbadie a vécu» (p.52) et propose à Flora Fontanges d'aller au musée afin d'observer des objets ayant servi aux premiers habitants du pays. Or, c'est davantage par le théâtre que par l'historiographie traditionnelle que Flora Fontanges entend redonner vie à Barbe Abbadie :

Elle rêve de s'approprier le cœur desséché de Barbe Abbadie, de l'accrocher entre ses côtes, de le rendre vivant à nouveau, comme un cœur de surcroît, de lui faire pomper un sang vermeil à même sa propre poitrine. [...] Il s'agit de mettre la main sur la bonne clef, et Flora Fontanges s'approprie sur-lechamp l'âme et le corps de Barbe Abbadie. [...] Flora Fontanges rayonne de la vie et de la mort de Barbe Abbadie. Elle devient puissante, envahissante, au comble de sa présence. Brille de tout son feu. Se penche vers Raphaël, par-dessus la table. C'est Flora Fontanges et ce n'est plus elle. (p. 52) 
Qualifiée de «merveilleuse» (p.53) par Raphaël, Flora Fontanges semble exercer une véritable fascination lorsqu'elle fait revivre une femme du passé. Plus encore, le texte suggère que cette manière d'accéder au passé de Flora Fontanges la rend puissante, lui donne une sorte de pouvoir associé à la lumière (" rayonner », «briller», «feu ») et, par le fait même, à la vie. Et lorsque Flora Fontanges incarne un nom de femme, ce sont toutes ses facultés intellectuelles qui sont mises à contribution. Le contraste entre la méthode de Raphaël (associée aux artefacts, aux musées, au découpage chronologique, bref à quelque chose d'achevé qui n'est plus actualisé par le présent) et celle de Flora Fontanges (associée à la lumière, au théâtre, à la subjectivité, à la vie) est frappant. Plus encore, le texte semble prendre parti pour la méthode de Flora Fontanges qui, par des moyens jugés moins scientifiques, parvient à un résultat dont la puissance dépasse de loin celle de l'historiographie. D'ailleurs, les deux versions des personnages, bien distinctes l'une de l'autre au début du roman, tendent à se confondre et à s'entremêler au fur et à mesure que Raphaël entre dans le jeu de Flora Fontanges.

\section{La quête de l'immémoire collective}

Les personnages féminins, dans l'œuvre romanesque d'Anne Hébert, n'apparaissent donc pas a priori comme des historiennes. Pourtant, si le personnage masculin est celui qui consigne le passé, la femme est celle qui le conserve pour le rappeler au présent : son champ de connaissance est bel et bien mémoriel, en ce sens que la mémoire n'est pas un contenu, mais une forme. Le passé, sous la forme du souvenir, est le contenu qu'elle organise. Malgré cette différence, les références au passé 
chez les personnages féminins d'Anne Hébert ont souvent été analysées par le biais de l'historiographie québécoise traditionnelle. Certains chercheurs, comme Éric Falardeau, affirment que le but d'Anne Hébert serait

[d']éliminer la faute originelle imputée à la collectivité, la conquête anglaise, pour retrouver le paradis perdu, Le premier jardin de la colonie. [...] La conquête anglaise incarne, à la lumière de cette déduction, le péché originel, la faute qui pèse sur la conscience historique des Canadiens français et à laquelle Flora veut à tout prix se soustraire. (p. 558)

Ainsi, selon Falardeau, le personnage de Flora évoluerait dans les rues de Québec avec la mémoire de la Nouvelle-France dans le but de nier la conquête anglaise et de faire revivre le temps de la colonie française sur la ville. Pourtant, rien n'est moins certain. Un tel angle d'approche nous semble même négliger tout un versant du rapport réflexif qu'entretient le personnage féminin au passé.

Raymond Côté souligne quant à lui que «l'interprétation de la poésie hébertienne suit les principes de Jung » (p. 91) par son symbolisme. En effet, sans vouloir tomber dans une analyse psychocritique, il nous semble que la théorie jungienne de la psychologie analytique peut jeter un regard pertinent sur ce mouvement mémoriel que l'on trouve dans Le Premier Jardin sans briser sa plurivocité. Jung distingue plusieurs catégories, voire plusieurs étapes de la psyché, le Moi, le conscient, mais dans le cas qui nous intéresse, les trois niveaux suivants: l'inconscient personnel, l'inconscient collectif et l'inconscient archaïque, ce dernier étant une partie de l'inconscient collectif qui ne peut être connu et que, transposé à la question mémorielle, on peut qualifier d'immémoire. 
Notre hypothèse serait que le personnage féminin de Flora Fontanges pense et articule le passé d'une manière différente, c'est-à-dire qu'elle cherche plutôt à aller au-delà du passé collectif historique (symbolisé par Raphaël) pour remonter à une forme d'immémoire collective. En fait, contrairement à Raphaël, le personnage de Flora Fontanges ne se contente pas de connaître l'histoire, mais opère une véritable réflexion autour du passé, qu'elle articule en trois niveaux intrinsèquement liés : (1) le passé personnel, (2) le passé collectif historique et (3) le passé collectif mythologique (que nous associons à l'inconscient archaïque de Jung).

Flora Fontanges ne cherche pas à éliminer la conquête anglaise comme l'affirme Falardeau (dans tout le roman, on trouve seulement deux mentions de la Conquête, laquelle n'est pas un enjeu majeur de l'œuvre), mais à se soustraire à ses propres souvenirs personnels par l'évocation des femmes du passé :

Flora Fontanges dont la mémoire est étrange et la concerne plus ou moins tant la peur de se compromettre lui fait puiser dans les souvenirs des autres, pêle-mêle, avec les siens propres afin qu'ils soient méconnaissables. (Hébert, p. 120)

C'est bien le premier niveau (le passé personnel) qui est ici nié par le personnage. Flora évite tous les lieux qui pourraient lui évoquer son enfance personnelle, tant le site de l'orphelinat que celui de la maison de sa famille adoptive. Le texte mentionne à plusieurs reprises que Flora Fontanges craint d'être confinée à un seul nom, celui de Pierrette Paul, qui était le nom qu'elle portait avant son adoption par les Éventurel, lorsqu'elle habitait à l'orphelinat. Le nom de l'enfance, auquel elle associe un rôle socio-construit, "risquait de la jeter dans le désespoir. N'être soi que toute sa vie, sans jamais pouvoir changer, être Pierrette 
Paul toujours, sans s'échapper jamais, enfermée dans la même peau, rivée au même cœur, sans espérance de changement» (Hébert, p. 63). Flora Fontanges se révolte contre un destin préfabriqué par des critères socio-historiques :

Éclater en dix, cent, mille fragments vivaces; être dix, cent, mille personnes nouvelles et vivaces. Aller de l'une à l'autre, non pas comme on change de robe, mais habiter profondément un autre être avec ce que cela suppose de connaissance, de compassion, d'enracinement, d'effort d'adaptation et de redoutable mystère étranger. (p. 64)

D'ailleurs, à chaque étape de sa vie correspond un nouveau nom: Pierrette Paul, Marie Éventurel, Flora Fontanges. Le masque de théâtre est l'outil qui lui permet de se libérer du particulier pour s'incarner dans l'universel, mais cet universel se joue par le biais du mythe.

À cet effet, si le premier niveau, le passé personnel, est nié par Flora Fontanges, le deuxième, le passé collectif historique, n'apparaît pas comme une finalité dans la quête mémorielle du personnage, mais comme un simple tremplin vers le troisième niveau, le passé collectif mythologique. L'exemple le plus explicite est sans doute celui de l'évocation du premier jardin de la colonie :

Est-ce donc si difficile de faire un jardin, en pleine forêt, et de l'entourer d'une palissade comme un trésor? Le premier homme s'appelait Louis Hébert et la première femme, Marie Rollet. Ils ont semé le premier jardin avec des graines venues de France. Ils ont dessiné le jardin d'après cette idée de jardin, ce souvenir de jardin, dans leur tête, et ça ressemblait à s'y méprendre à un jardin de France, jeté dans la forêt du Nouveau Monde. [...] Quand le pommier, ramené d'Acadie par M. de Mons, et transplanté, a enfin donné ses fruits, c'est devenu le premier de tous les jardins du monde, avec Adam et Ève devant le pommier. Toute l'histoire du monde s'est mise à 
recommencer à cause d'un homme et d'une femme plantés en terre nouvelle.

Flora Fontanges est saisie par ce commencement des temps qu'il y eut dans la ville et dont lui parle Raphaël. Il s'anime. Il croit que la vie ancienne est à rattraper dans toute sa fraîcheur, grâce à l'Histoire. Elle dit que le temps retrouvé, c'est du théâtre, et qu'elle est prête à jouer Marie Rollet sur-le-champ. (Hébert, p. 76)

On voit bien ici que le personnage de Flora Fontanges, dans son parcours mémoriel, ne s'arrête pas au passé historique (le jardin de Marie Rollet et de Louis Hébert, premiers colons de Québec et ancêtres de la romancière), mais se sert de cet élément comme pivot vers le passé mythologique (le jardin d'Éden). À preuve, loin de se limiter à la colonie française et à la conquête anglaise, le texte mentionne un objectif fort différent :

remonter à la source du monde, retrouver la fraternité première avec les plantes et les animaux, ne plus croire à la séparation orgueilleuse de l'homme et de la femme d'avec le reste de la création. (p. 71)

Cette dernière remarque n'est pas banale : le passé historique, par opposition au passé mythique, est associé à l'inimitié et à la division dont il consigne et répète le souvenir. Lorsque Flora Fontanges interprète Marie Rollet, elle semble parfaitement consciente du glissement mythologique qu'elle opère :

En réalité, c'est d'elle seule qu'il s'agit, la reine aux milles noms, la première fleur, la première racine, Ève en personne (non plus seulement incarnée par Marie Rollet, épouse de Louis Hébert), mais fragmenté en mille frais visages, Ève dans toute sa verdeur multipliée, son ventre fécond, sa pauvreté intégrale. (p. 99)

L'archétype d'Ève, parce qu'il incarne «mille frais visages » de femmes plutôt que d'exclure, est vécu comme une vengeance contre l'histoire et ses oublis (l'oubli de Barbe Abbadie par 
exemple), en redonnant à la femme des assises symboliques, mythologiques. La différence est que l'universalité rend le mythe plus inclusif, plus représentatif de tous les particuliers, contrairement à l'histoire qui s'érige sur une sélection (et donc sur l'oubli) de quelques particuliers à partir desquels elle se prétend générale. Et la limite historique, ici l'arrivée des premiers colons en sol canadien et leur établissement, sert de tremplin mémoriel pour transiter vers le mythe.

C'est surtout ce dernier niveau — le passé mythologique - , il nous semble, qui fait que la lecture féminine du passé diffère de l'historiographie traditionnelle et échappe à ses outils d'analyse. Analyser l'œuvre d'Anne Hébert par l'angle de l'historiographie, c'est sans doute passer à côté de son apport le plus original à la lecture du temps. La mémoire collective — l'inconscient collectif chez Jung - dont Falardeau faisait la visée du Premier Jardin apparaît ici, il est vrai, comme un point traversé par l'axe (d'où sa pertinence dans la lecture de l'œuvre), elle mais n'en consiste cependant pas la finalité. Cette finalité, chez Anne Hébert, n'est nulle autre que l'inconscient archaïque, associé ici au mythe. Cet inconscient archaïque réfère à une période qui précède l'histoire, donc échappant à toutes traces écrites. C'est pourquoi le personnage de Flora, ne pouvant remonter par le factuel à cet inconscient archaïque, va d'abord s'attarder à sa toute première limite avec l'Histoire collective, c'est-à-dire les débuts de la colonie française. À preuve l'image du premier jardin, celui des premiers colons de Québec, Louis Hébert et Marie Rollet, nous l'avons vu, ne constitue pas, au final, l'archétype générateur : il ne sert que de prétexte à remonter vers le premier jardin biblique, celui d'Adam et Ève, le jardin d'Éden. Le personnage féminin de Flora Fontanges repense le rapport au passé en saisissant la religion catholique « non pas dans sa pratique quotidienne ou ses 
dogmes moraux, mais comme accès à un symbolisme universel » (Biron, Dumont et Nardout-Lafarge, p. 310).

\section{Conclusion}

Cette quête mémorielle de Flora Fontanges, tout comme la quête de Don Quichotte ou d'Emma Bovary, s'avère un échec. Elle ne parviendra pas à faire revenir le temps mythologique sur la ville, tout juste l'incarne-t-elle un certain temps. Raphaël ${ }^{2}$ parviendra à retrouver Maud, la fille de Flora, et celle-ci entraînera sa mère dans la ville moderne et nocturne de Québec : «[a]lors commence une visite de la ville comme Flora Fontanges n'en a jamais connue » (Hébert, p. 181). Les avenues pleines de monde dans la nuit chaude, des «enseignes qui clignotent» (p.181), des "discothèques [qui] surgissent au hasard» (p. 181), des «nuages de fumée» (p. 181) projettent Flora Fontanges dans «le bruit et la fureur de vivre» (p. 181), au son du rock, bien loin des ballades et de l'évocation de la Nouvelle-France. Cette dernière nuit a tout d'une épreuve initiatique pour Flora Fontanges, qui découvre le temps présent et met en échec sa quête mémorielle. Le retour de Flora à Québec se termine par un nouveau départ, mais cette fois, « [s]a mémoire éteinte ne pèse pas plus qu'une feuille morte» (p. 167)

\footnotetext{
2 Le choix du nom de Raphaël n'est d'ailleurs pas banal, comme l'indique le qualificatif d'archange utilisé à deux reprises dans le texte. L'archange Raphaël est le patron des voyageurs et la racine « Rapha » de son nom signifie en hébreu guérir. Dans le Deutérocanonique, Raphaël est envoyé pour accompagner Tobie dans son voyage et pour guérir la cécité de son père. Dans Le Premier Jardin, Raphaël accompagne Flora dans son exploration de la ville, l'aide à retrouver sa fille et à affronter son passé personnel.
} 


\section{Bibliographie}

BIRON, Michel, François Dumont et Élisabeth NARDouT-LAFARGE. (2007), Histoire de la littérature québécoise, Montréal, Boréal.

CôTÉ, Raymond. (1989), « Le Premier Jardin d'Anne Hébert ou le faux double dénoncé », American Review of Canadian Studies, vol. XIX, no 1 , p. 83-92.

DAUNAIS, Isabelle. (2008), Les Grandes Disparitions : essai sur la mémoire du roman, Saint-Denis, Presses universitaires de Vincennes.

FALARDEAU, Éric. (1997), «Fictionnalisation de l'histoire, Le Premier Jardin d'Anne Hébert», Voix et images, vol.XXII, no 3 (66), p. 557-568.

HÉBERT, Anne. (1988), Le Premier Jardin, Paris, Seuil.

JunG, Carl Gustav. (1996), Psychologie de l'inconscient, Paris, Georg éditeur, coll. « Livre de poche ». 


\section{Résumé}

Dans Le Premier Jardin d'Anne Hébert, Flora Fontanges marche dans les rues de Québec en compagnie du jeune Raphaël, un étudiant en histoire. C'est l'occasion, pour la romancière, de suggérer que le personnage masculin de Raphaël, lié à l'historiographie, et le personnage féminin de Flora, une comédienne, possèdent deux manières différentes d'interpréter le passé. Les références au passé dans l'œuvre d'Anne Hébert ont souvent été analysées par le biais de l'historiographie traditionnelle. Un tel angle d'approche, s'il est permis par le texte, nous semble toutefois négliger tout un versant du rapport réflexif qu'entretient le personnage féminin au passé, soit l'aspect mythologique.

\section{Abstract}

In Anne Hébert's Premier Jardin, Flora Fontanges walks in the streets of Quebec City with Raphaël, a young History student. This is the occasion, for the novelist, to suggest that the masculine character of Raphaël, associated to historiography, and the feminine character of Flora, an actress, have two different ways to analyse the past. The references to the past, in Anne Hébert's novels, have often been analysed by traditional historiography. If this perspective is justified by the text itself, it appears to us that it doesn't include an important aspect of the intellectual link that the feminine character has developed to the past, namely the mythological aspect. 